Technological University Dublin

DÜBLIN

ARROW@TU Dublin

2004-01-01

\title{
Application of ESPI-Method for Strain Analysis in Thin Wall Cylinders
}

\author{
David Kennedy \\ Technological University Dublin, david.kennedy@tudublin.ie \\ Zdravko Schauperl \\ Technological University of Dublin, zdravko.schauperl@fsb.hr \\ Seamus Greene \\ Technological University of Dublin, Seamus.Greene@tudublin.ie
}

See next page for additional authors

Follow this and additional works at: https://arrow.tudublin.ie/cieoart

Part of the Engineering Commons

\section{Recommended Citation}

Kennedy, D. et al. (2004) Application of ESPI-Method for Strain Analysis in Thin Wall Cylinders. Optics and Lasers in Engineering, vol.41, pp. 927, 585-594. doi:10.1016/S0143-8166(03)00015-0

This Article is brought to you for free and open access by the Centre for Industrial and Engineering Optics at ARROW@TU Dublin. It has been accepted for inclusion in Articles by an authorized administrator of ARROW@TU Dublin. For more information, please contact arrow.admin@tudublin.ie, aisling.coyne@tudublin.ie, gerard.connolly@tudublin.ie.

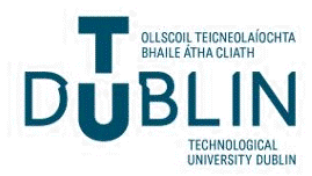




\section{Authors}

David Kennedy, Zdravko Schauperl, Seamus Greene, and Emilia Mihaylova 


\title{
Application of ESPI-method for strain analysis in thin wall cylinders
}

\author{
D.M. Kennedy ${ }^{1}$, Z. Schauperl ${ }^{1}$, S. Greene ${ }^{1}$, E.M. Mihaylova ${ }^{2}$ \\ ${ }^{1}$ Faculty of Engineering, Dublin Institute of Technology, Bolton Street, Dublin 1, Ireland \\ ${ }^{2}$ Centre for Industrial and Engineering Optics, School of Physics, Dublin Institute of Technology, \\ Kevin Street, Dublin 8, Ireland
}

\begin{abstract}
The Centre for Industrial and Engineering Optics, DIT, Ireland, have recently developed and applied new optical techniques for the measurement of mechanical strain, one of which was based on electronic speckle pattern interferometry (ESPI). The accuracy of this optical technique when used on flat surfaces is well established. In this research the technique is tested on curved surfaces, and results are compared with the theoretical hoop strain as predicted by the bi-axial strain equation modified for the thin cylinder, and by those obtained by electrical resistance strain gauge (ERSG) undoubtedly the principal method of measuring mechanical strain. For testing procedures, a special unit was designed and produced for holding and loading the thin cylinder. Also the basic equation for hoop strain by ESPI for flat surfaces was modified for applications on curved surfaces.

Thin cylinder hoop strain obtained by ESPI and calculated by a modified equation show remarkably good correlation to the predicted theoretical value as well as to the results obtained by ERSG. Since the ESPI theory was originally developed for use on flat surfaces, there are reasonable grounds for further investigating this relationship especially where curved surfaces are involved.
\end{abstract}

Keywords: ESPI; ERSG; Hoop strain; Curved surfaces

\section{Introduction}

The principal method of measuring mechanical strain is undoubtedly electrical resistance Strain gauge (ERSG). Therefore, any new technique for measuring mechanical strain is usually benchmarked against it. The Centre for Industrial and Engineering Optics at DIT, Ireland, have developed and applied new optical techniques for measuring mechanical strain, based on electronic speckle pattern interferometry (ESPI). The accuracy of this optical technique when used on flat surfaces was well established, but its use on curved surfaces was untested. Therefore, this research is directed to investigate the applicability of ESPI for measuring strain in a thin cylinder. The same strain is measured by ERSG, and both results were compared with predicted mechanical strain values obtained from theory. The values of the mechanical strains were evaluated using Mohr's circle theory, while the optical strains were obtained from the basic equation of ESPI modified for curved surfaces 


\section{Electronic speckle pattern interference}

In ESPI a speckle pattern is formed by illuminating of the surface of the object to be tested with two collimated wave fronts formed by an interferometer having laser light as its source. A schematic of the system is shown in Fig. 1.

The wave fronts, $u_{1}$ and $u_{2}$; are symmetrically inclined at an angle $\alpha$ to the surface normal to the test object in the x-plane. The unloaded test object is imaged by a CCD video camera directed along the z-axis and in the plane of the collimated wave fronts. These wave fronts are scattered at the surface of the object and form two separate speckle patterns that interfere in the film plane of the CCD camera [1].

The CCD camera captures this interference pattern, which is then processed by the ESPI test software and is stored as a reference pattern on the computer.

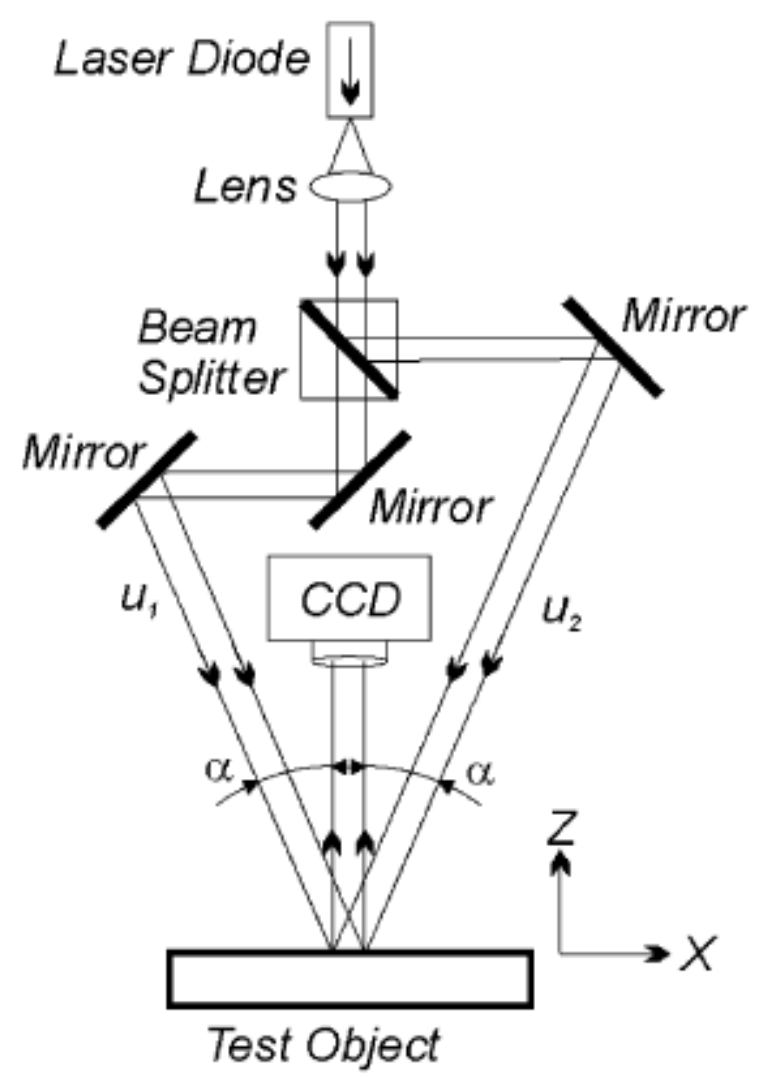

Fig. 1. Arrangement of interferometer to measure displacement in the direction of x-axis.

When the object is subsequently loaded, the test area undergoes deformation and the collimated wave fronts are again scattered, resulting in a change in the speckle patterns. This in turn causes a change in the interference pattern, which is again imaged on the faceplate of the CCD camera and stored in memory. The ESPI test software is then used to process the two stored interference patterns so as to display the resulting interferogram of alternate light and dark fringes on video monitor. These fringes represent contours of equal displacement in the direction of the $\mathrm{x}$-axis, [2]. 
From ESPI theory, the fringe spacing is given by

$$
\Delta x=\frac{d}{n}=\frac{\lambda d}{2 \Delta d \sin \alpha} \text {. }
$$

From which the displacement $\Delta \mathrm{d}$ is obtained [3]:

$$
\Delta d=\frac{n \lambda}{2 \sin \alpha}
$$

where $\lambda$ is the wavelength of the laser light, $d$ the reference length measured on the surface along the $\mathrm{x}$-axis, $\Delta \mathrm{d}$ the displacement of the reference length in the direction of the $\mathrm{x}$-axis, $\mathrm{n}$ the number of fringes forming the interferogram and the angle of inclination of coherent wave front to surface normal in z-plane.

The interferogram consists of alternate light and dark fringes, called correlation fringes, as the fringes are produced by correlating the intensities of the resultant speckle patterns recorded before and after displacement. One example of this is shown in Fig. 2. The fringes are of similar appearance to moire fringes and represent contours of equal displacement, with each fringe resulting from a displacement of the order of half a wavelength of the laser light source used in the interferometer. Hence the interferogram is a representative of the superficial displacement or deformation of the test object, and the number of fringes indicates the degree or extent of the displacement. The geometry of the fringes also provides an indication of the geometry of deformation. Where the fringes are evenly spaced and of similar shape and size, the underlying surface is uniformly strained. Where the fringes are distorted or are of uneven size the underlying surface is not uniformly strained, thereby indicating a surface or subsurface structural abnormality or defect.

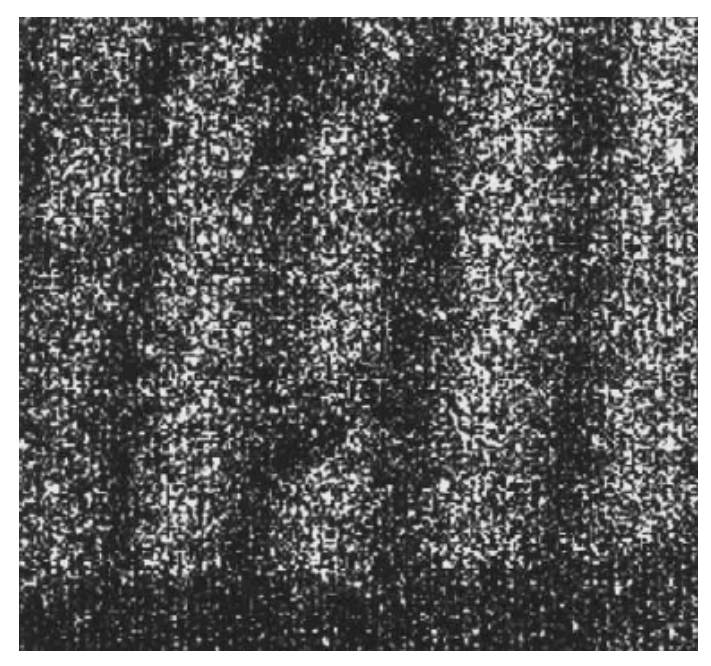

Fig. 2. Example of interferogram.

In an in-plane interferometer, when the test object illuminated by the two collimated beams is displaced by a distance $\Delta \mathrm{d}$ in the $\mathrm{x}$-plane, the relative phase change of the two beams is given by [4] 


$$
\Delta \phi=\frac{4 \pi \Delta d \sin \alpha}{\lambda}
$$

where $\lambda$ is the wavelength of the laser light source of the interferometer.

\section{Experimental}

\subsection{Thin cylinder apparatus}

A diagram of the thin cylinder apparatus is shown in Fig. 3. A thin walled tube (A), contained two pistons (B) and (C), is threaded externally at both ends to receive end stops (D). Piston (B) is located axially within the tube by end stop (D). This piston extends beyond the end of the tube and is drilled to suit a four-way adaptor, to which is fitted a quick release self-sealing coupling, a pressure gauge and a spring loaded pressure relief valve. Oil from this valve returns to the pump reservoir connecting through flexible tube. A high-pressure flexible rubber hose connects a hand operated pressure pump through a large screw valve to the cylinder unit. This valve is used to lock the desired test pressure in the cylinder.

The cylinder unit which is resting on the four pins (R) is supported in a frame (M) and located axially by a fixed and adjustable stop (O). When the adjustable stop is screwed out the pressurised oil in the cylinder forces piston $(C)$ against the fixed end stop and the entire axial load is taken on the frame, thus relieving the cylinder of all longitudinal stress. Pure axial load transmission from cylinder to frame is ensured by the hardening steel balls (S) situated each end of the cylinder. The complete apparatus is mounted on a bench and has two modes of operation, with open ends (for determination of hoop strain) and with closed ends (for determination of longitudinal hoop strain).

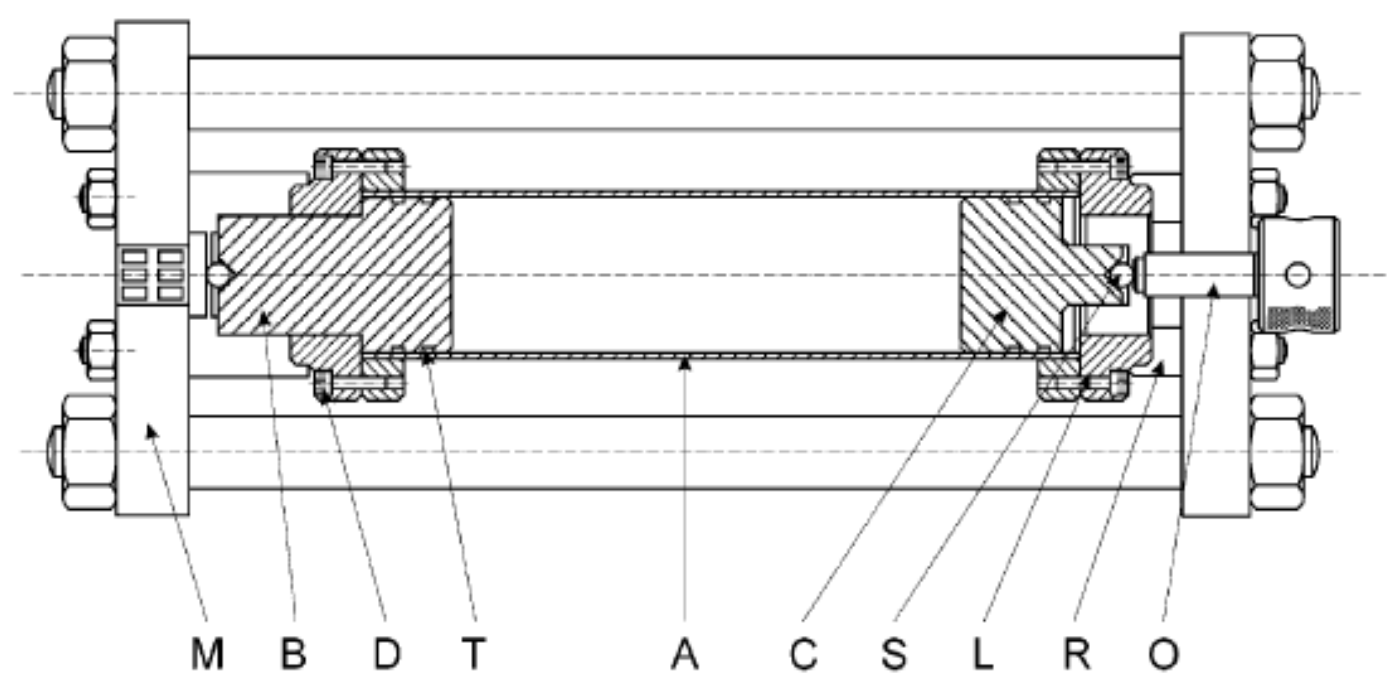

Fig. 3. Sectional plan view of thin cylinder unit. 


\subsection{Deformation of a thin walled cylinder}

A Thin Walled Cylinder is defined by

$$
\frac{D}{t} \gg 20,
$$

where $D$ is the bore diameter, and the wall thickness.

The circumference of any cylinder is proportional to its diameter; therefore, the circumferential or hoop strain is directly related to the diametrical strain as shown in Fig. 4.

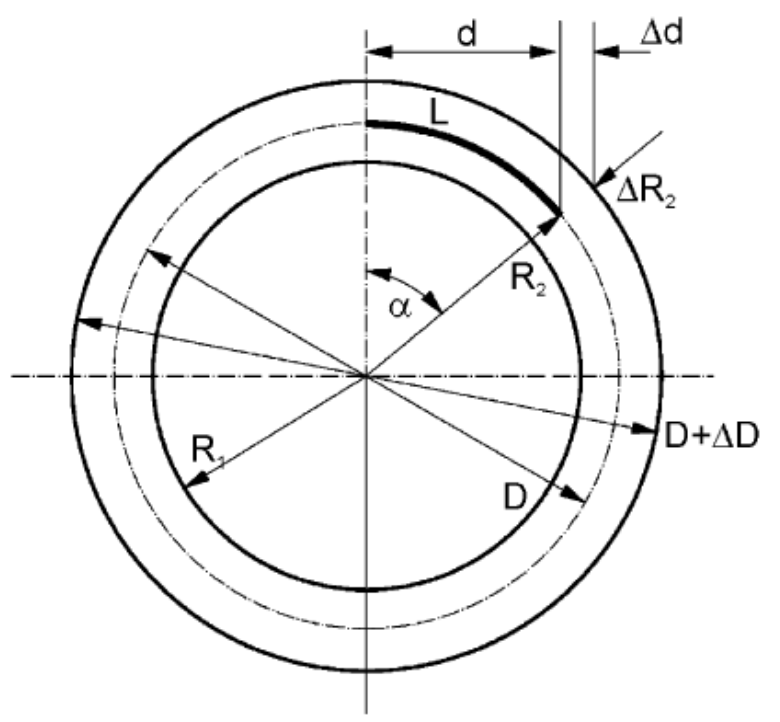

Fig. 4. Geometry of deformation of thin walled cylinder.

From Fig. 4:

$$
\begin{aligned}
& \varepsilon_{\mathrm{C}}=\frac{\Delta D}{D}=\frac{2 \Delta R_{2}}{2 R_{2}}=\frac{\Delta R_{2}}{R_{2}}, \\
& \sin \alpha=\frac{d}{R_{2}}=\frac{\Delta d}{\Delta R_{2}}, \\
& \Delta R_{2}=\frac{\Delta d}{d} R_{2}=\frac{n \lambda}{2 \sin \alpha} \frac{R_{2}}{d}
\end{aligned}
$$

and

$$
\varepsilon_{\mathrm{C}}=\frac{\Delta R_{2}}{R_{2}}=\frac{n \lambda}{2 \sin \alpha} \frac{R_{2}}{d} \frac{1}{R_{2}} .
$$


The hoop strain by ESPI is

$$
\varepsilon_{\mathrm{C}}=\frac{n \lambda}{2 d \sin \alpha} \text {. }
$$

This equation works for ESPI on flat surfaces. As the surface of the test cylinder was curved there was a difference between the horizontal length of the projected area as captured on the faceplate of the CCD camera, $d$; and its actual curved length on the circumference of the cylinder, $L$. This actual length of surface, according to the geometry, is

$$
L=\alpha \frac{2 \pi}{360} R_{2}
$$

For application of the ESPI method on curved surfaces, reference length $d$ should be replaced with the real surface length, L: The modified equation for hoop strain by ESPI on curved surfaces is

$$
\varepsilon_{\mathrm{C}}=\frac{n \lambda}{2 \sin \alpha} \frac{360}{\alpha 2 \pi R_{2}}
$$

For a given arrangement of the in-plane interferometer, where the values of $d ; \lambda$ and $\alpha$ are fixed, the hoop strain is evaluated by multiplying the fringe order $\mathrm{n}$ by a constant $\mathrm{k}$ :

$$
\varepsilon_{\mathrm{C}}=k n,
$$

where the constant $\mathrm{k}$ is

$$
k=\frac{360 \lambda}{4 \alpha \pi R_{2} \sin \alpha} .
$$

In the arrangement used in the present test configuration, the values of the above variables are:

$\lambda=785 \times 10^{-6} \mathrm{~mm}, \alpha=38^{\circ} ; \mathrm{R}_{2}=38.1 \mathrm{~mm}$. Inserting these values in Eq. (13) it is possible to calculate the constant $\mathrm{k}$ :

$$
k=\frac{360 \times 785 \times 10^{-6}}{4 \times 38 \times \pi \times 38.1 \sin 38}=0.00002523 .
$$

Constant $\mathrm{k}$ is $25 \mu \varepsilon$ per fringe.

The strain of the thin cylinder for different internal pressures could be calculated by multiplying the kvalue with the recorded number of fringes on the interferogram, $n$.

\subsection{Measurement preparation}

The strain of the thin cylinder was measured simultaneously by ERSG and ESPI for each internal pressure, and it was also calculated by using the theory of thin cylindrical shells. 


\subsubsection{Bonding of rosette strain gauge}

To measure the hoop and axial strains on the surface of the thin cylinder a stacked rosette strain gauge was bonded onto the outer surface of the cylinder, using cynoacrylate. Lead wires were soldered to each gauge element using the 3-wire 1/4bridge connection format, and each gauge was then connected individually to the switch and balance unit attached to the strain indicator.

As shown in Fig. 5, the gauge was located close to the area where the interferometer was to be positioned so that the strain gauge and optical measurements would be taken from the same cylinder section.

\subsubsection{Set up of ESPI in-plane interferometer}

The interferometer unit was set up in the vertical plane directly above a section of the thin cylinder, which had been coated with a matt white paint, as a suitable background on which the wave fronts could be observed. The mirrors of the interferometer were individually adjusted so that the collimated or flat wave fronts or beam have the same brightness or intensity. This process was one of trial and error, as each wave front or beam was adjusted in turn until they both appeared to be of equal brightness or intensity. At this point the optical distances from the mirrors and the beam splitter to the test area were measured using a scale rule. Once set up these elements were fixed for the duration of testing. Using simple trigonometry, the optical distance measurement were used to evaluate $a$; the angle between the surface normal in the z-plane and the incident wave fronts. The ESPI software was set up on the computer connected to the CCD video camera on the interferometer, which was focused on the test area.

The length of the test area, $d$; was measured on the monitor by placing a scale rule over the test area and reading this dimension on the computer monitor. This method of measuring the test area allows for any magnification involved in the optical system, since the field of view of the camera is indicated directly by the length of the scale rule visible on the computer screen. In all tests carried out, this dimension was measured as $19 \mathrm{~mm}$, angle of incident beams $(\alpha)$ is $38^{\circ}$ and wavelength of the laserlight, $(\lambda)$ was $785 \times 10^{-6} \mathrm{~mm}$. The optical system was then ready for testing.

\subsubsection{Theory of thin cylindrical shells}

From the theory of thin cylindrical shells [5], it is possible to theoretically determine of the hoop strain by using the equation:

$$
\varepsilon_{\mathrm{C}}=\frac{P D(2-v)}{4 t E},
$$

where $\mathrm{P}$ is the internal pressure, $\mathrm{D}$ the cylinder bore, $\mathrm{U}$ the Poisson's ratio, $\mathrm{E}$ the Young's modulus and $t$ the cylinder wall thickness. 


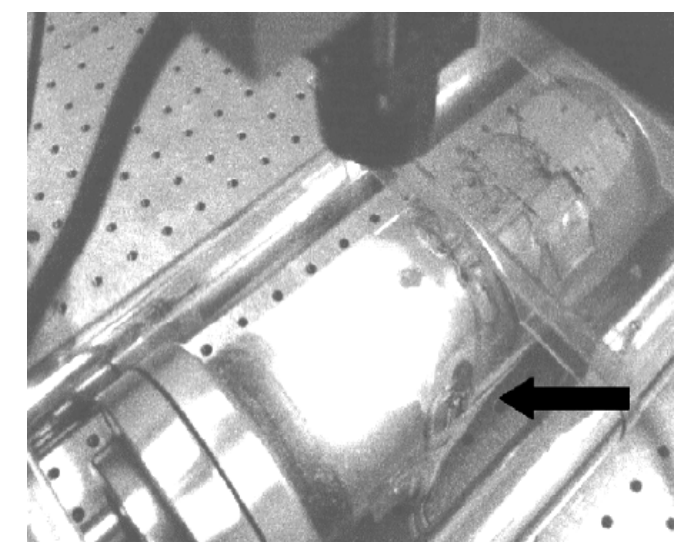

Fig. 5. The gauge location, arrow.

\subsection{Test procedure}

The cylinder, with diameter $76.2 \mathrm{~mm}$, made of steel with Poisson's ratio 0.274 and Young's modulus of $226 \mathrm{GN} / \mathrm{m}^{2}$, was pressurised to 28 bar and the strain gauge readings were recording for each of the three elements of the rosette.

The recording of the reference or initial speckle pattern was than initiated by clicking the mouse button on the start icon of the ESPI screen. At this point the hydraulic pressure release valve was manually activated thereby unloading the pressure in the cylinder. This relaxation of the internal pressure in the cylinder brought about a corresponding relaxation in the cylinder's deformation, and it returned to its original unstrained condition. After the gauge pressure had reached zero, the ESPI recording was ended by clicking on the stop icon on the ESPI screen.

The interferogram resulting from the subtraction of the two speckle patterns was then displayed on the screen. Since the ESPI test software allowed the interferograms from repeated tests to be compared with each other, at each pressure only the optimum interferogram was selected for analysis and stored as a picture files on a computer. This procedure was repeated several times at each pressure interval from 4 to 28 bar in the first series of the tests, and from 2 to 29 bar in the second series. Furthermore, these two series were repeated, resulting in an overall total of 171 files being recorded. From these files a selection of interferograms from each of the two series of tests was downloaded for subsequent analysis.

\section{Results}

Full test results for both series of tests showing:

* optical strain values obtained from the ESPI equation for hoop strain,

* the theoretical hoop strain as predicted by the bi-axial strain equation modified for thin cylinder and

* experimental values of the reduced rosette gauge strain readings of hoop strain are presented in Tables 1 and 2 . 
Table 1

Test results for the first tests, for internal pressure from 2 to 29 bar

\begin{tabular}{lccc}
\hline Cylinder pressure, $p$ (bar) & \multicolumn{2}{l}{ Hoop strain, $\mu \varepsilon$} & ESPI \\
\cline { 2 - 4 } & Theoretical & ERSG & 10 \\
\hline 2 & 9 & 7 & 25 \\
5 & 23 & 19 & 44 \\
8 & 37 & 32 & 58 \\
11 & 50 & 46 & 73 \\
14 & 64 & 59 & 88 \\
17 & 78 & 73 & 98 \\
20 & 92 & 87 & 113 \\
23 & 105 & 101 & 125 \\
26 & 119 & 115 & 138 \\
29 & 133 & 129 & 0.998281 \\
Correlation & & 0.999853 & $6.8<17$, accept \\
Chi test for goodness of fit & & $2.93<17$, accept & \\
\hline
\end{tabular}

Table 2

Test results for the second tests, for internal pressure from 4 to $28 \mathrm{bar}$

\begin{tabular}{lccc}
\hline Cylinder pressure, $p$ (bar) & \multicolumn{2}{l}{ Hoop strain, $\mu \varepsilon$} & ESPI \\
\cline { 2 - 4 } & Theoretical & ERSG & 21 \\
\hline 4 & 18 & 16 & 38 \\
7 & 32 & 29 & 55 \\
10 & 46 & 42 & 70 \\
13 & 60 & 55 & 83 \\
16 & 73 & 69 & 95 \\
19 & 87 & 82 & 108 \\
22 & 101 & 96 & 120 \\
25 & 115 & 109 & 131 \\
28 & 128 & 124 & 0.997988 \\
Correlation & & 0.999879 & $7.4<15.5$, accept \\
Chi test for goodness of fit & & $2.23<15.5$, accept & \\
\hline
\end{tabular}

\section{Conclusions}

The aim of this work was to investigate the application of ESPI technique for determination of hoop strain on curved surfaces. This method was tested on the thin wall cylinder pressurised up to 29 bar. The surface of the test cylinder was curved and so there was a difference between the horizontal length of the projected area as captured on the faceplate of the CCD camera and its actual curved length on the circumference of the cylinder. Therefore, the main equation for hoop strain by ESPI on flat surfaces was modified. In the new Eq. (11), the reference length d was replaced with the real surface length, L.

Comparison of ESPI results with the theoretical hoop strain as predicted by the biaxial strain equation, and by those obtained by ERSG, undoubtedly the principal method of measuring mechanical strain, shows that the ESPI optical strain measuring technique was found to be an accurate method for measuring hoop strain in the thin cylinder. 
The small deviation of the ESPI results could be attributed to a variety of factors, from the measurement of the interferometer's optical elements, to the difficulty during measuring the fringes since the number of fringes measured was small. Also, when the surface of the object is not flat, it can be shown that the fringes may have sensitivity to out-of-plane movement or displacement [6].

However, this research shows remarkably good correlation of ESPI results to the predicted theoretical value and to the results obtained by ERSG, and since the ESPI theory was developed for use on flat surfaces there are reasonable grounds for further investigating this relationship involving a new equation where curved surfaces are involved. So further research on that area is directed to investigate the applicability of the same method for strain analysis on thick wall cylinders.

\section{References}

[1] Sirohi RS. Speckle metrology. New York: Marcel Decker, 1993.

[2] Gasvik KJ. Optical metrology. New York: Wiley, 1995.

[3] Jones R, Wykes C. Holographic interferometry. Cambridge: Cambridge University Press, 1983.

[4] Gasvik KJ. Optical metrology. New York: Wiley, 1995.

[5] Urry SA, Turner PJ. Strength of materials and mechanics of solids, 1974.

[6] Butters JN, Leendertz JA. A double exposure for speckel pattern interferometry. J Phys E: Sci Instrum 1971: 227-9. 